\title{
Requerimientos energéticos y proteicos \\ estimados por calorimetría indirecta y nitrógeno \\ urinario en pacientes con quemadura \\ o pancreatitis aguda
}

\author{
Energy and protein requirements \\ estimated by indirect calorimetry and \\ urine urea nitrogen in burn and \\ acute pancreatitis patients
}

\begin{abstract}
The pathological states of burn injuries and acute pancreatitis are associated with hypermetabolic and hypercatabolic states. The objective of the study was to determine energy and protein requirements in these conditions. A transversal analytical study was applied to 18 patients: 10 with mild burn injuries in $<5 \%$ body surface and 8 with acute pancreatitis. Anthropometric measurements were taken (weight, height, BMI, skin folds), as well as resting metabolic rate through indirect calorimetry (RMR IC), protein catabolism using 24-hour urine urea nitrogen (UUN), and trauma factor (RMR IC/BMR Harris-Benedict equation). The sample was made up of $72.2 \%$ men and $27.8 \%$ women. The study of burn and acute pancreatitis patients was carried out on the average 16.1 \pm 13.0 SD and $8.8 \pm 2.0$ SD days after the onset of the disease, respectively. There were no statistically significant differences in RMR IC since mean energy requirements were $24.4 \mathrm{kcal} / \mathrm{kg} / \mathrm{d}$ and $23.2 \mathrm{kcal} / \mathrm{kg} / \mathrm{d}$ in patients with burns and acute pancreatitis, respectively, for both sexes ( $p>0.05$ ). Total energy expenditure (TEE) was not significant $(p>0.05)$. Acute pancreatitis and burn patients showed mild catabolism and their protein requirement was 1,19g/ $\mathrm{kg} / \mathrm{d}(\mathrm{p}>0.05)$. The trauma factor in acute pancreatitis patients was 0,91 ( $p>0.05$ ). In conclusion, patient energy requirement was lower than reported or recommended values in both pathologies under study, whereas protein requirement was lower in burn patients. Key words: Protein requirement, urine urea nitrogen, acute pancreatitis, burn, energy requirement.
\end{abstract}

Marcela Ruiz De la F. (1) Nataly Sepúlveda Ch. (2) Alejandra Rodriguez F. (1)

(1) Departamento de Nutrición y Salud Pública, Facultad de Ciencias de la Salud y de los Alimentos, Universidad del Bío-Bío, Chillán, Chile. (2) Centro de Salud familiar Cartagena, Provincia de San Antonio, Chile.

Dirigir la correspondencia a: Profesora

Marcela Ruiz De la Fuente Nutricionista Magister en Nutrición Clínica Adulto Departamento de Nutrición y Salud Pública Universidad del Bío-Bío, sede Chillán Teléfono: (56-42) 2463229 Email:marcelaruiz@ubiobio.cl

Este trabajo fue recibido el 27 de Agosto de 2013 y aceptado para ser publicado el 12 de Enero de 2014.

\section{INTRODUCCIÓN}

En la clínica nutricional, es habitual considerar que pacientes con pancreatitis aguda o quemaduras menores, padezcan estados hipermetabólicos $(1,2)$ y/o hipercatabólicos (3), influenciados entre otros factores, por el grado de severidad de la patología presente (4).

Al estimar los requerimientos energéticos totales del paciente, mediante ecuaciones predictivas, es habitual agregar en el cálculo, además del factor de actividad física, el factor trauma o de corrección $(1,2)$, el cual considera el porcentaje del aumento de la tasa metabólica basal presente, situación observada en algunos estados patológicos y de manera variable.
Recientemente se conoce con mayor precisión el gasto energético real de los pacientes, lo que ha provocado la reducción de los factores de corrección energética. Estos factores, pueden llevar a sobrestimar el requerimiento energético del paciente, lo cual no implica disminuir las pérdidas nitrogenadas presentes, sino facilitar los depósitos de grasa y la aparición de complicaciones metabólicas secundaria a la sobrealimentación (5).

En el estado hipercatabólico, se ha descrito una considerable destrucción proteica y por ende un aumento de los requerimientos de este nutriente en el paciente, sin embargo, aportes proteicos muy elevados, podrían facilitar el aumento 
de la degradación proteica neta e incrementar la tasa de catabolismo (6).

En relación a lo anterior los objetivos de la investigación fueron: determinar los requerimientos energéticos y proteicos de pacientes con pancreatitis aguda o quemaduras menores; y analizar la influencia de la patología en los resultados. Estableciéndose como hipótesis del estudio que el requerimiento energético y proteico es mayor en el paciente quemado.

\section{SUJETOS Y MÉTODO}

El estudio fue analítico, de corte transversal. Se estudiaron 18 pacientes de ambos géneros del Hospital Clínico Herminda Martín de Chillán. De ellos, 8 padecían pancreatitis aguda y 10 sufrían quemaduras menores al 5\% de la superficie corporal. En los pacientes con pancreatitis aguda, la distribución porcentual de acuerdo a los criterios de gravedad de Balthazar (7) fue la siguiente: $12,5 \%$ clasificó con pancreatitis tipo A, 25\% tipo C, $37,5 \%$ con tipo D y un $25 \%$ con tipo E. Respecto al índice de severidad (8) el 62,5\% clasificó en severidad baja, el 12,5\% en media y el $25 \%$ en alta. En estos pacientes el estudio se realizó en promedio a los $8,8 \pm 2,0$ DS días, posteriores al inicio de los síntomas de la enfermedad.

La distribución porcentual de pacientes quemados de acuerdo a la clasificación de severidad, según American Burn Association (9), mostró que un 10\% de ellos presentó quemadura grave, un 50\% quemadura moderada y un $40 \%$ quemadura leve. El estudio en los pacientes quemados se realizó en promedio a los 16,1 \$13,04 DS días, posteriores al inicio de la enfermedad.

De los 18 pacientes estudiados, todos se encontraban recibiendo sólo alimentación vía oral. El estudio contó con la aprobación del Comité de Ética del hospital y con el consentimiento informado de los pacientes.

\section{MEDICIONES ANTROPOMÉTRICAS}

Se determinó el peso corporal a través de una balanza marca SECA modelo 713, con escala de $2 \mathrm{~kg}$ a $130 \mathrm{~kg}$ y precisión de 0,2 kg; talla corporal a través de un tallímetro con graduación mínima de $1 \mathrm{~mm}$; pliegues cutáneos (bícipital, trícipital, subescapular y supraíliaco), mediante un plicómetro marca Lange con sensibilidad de $1 \mathrm{~mm}$, graduación de 0-67 $\mathrm{mm}$. (10-12). Se determinó el índice de masa corporal o IMC $\left(\mathrm{Kg} / \mathrm{m}^{2}\right)(11)$, utilizando los criterios establecidos por la Organización Mundial de la salud (OMS).

De la sumatoria de los pliegues cutáneos y utilizando la ecuación de Durnin y Womersley (13), se obtuvo el porcentaje de la masa grasa (MG) y, por diferencia, el porcentaje de la masa libre de grasa (MLG).

En los diferentes cálculos se utilizó el peso corporal real del paciente, salvo aquellos con estado nutricional de obesidad, donde se utilizó el peso corporal ajustado (14). Peso ajustado $=(($ peso actual-peso ideal $) \times 0,25)+$ peso ideal; considerando que el $25 \%$ del exceso de peso corporal está constituido por masa magra metabólicamente activa, y el 75\% restante corresponde a tejido adiposo (15).

\section{ENCUESTA NUTRICIONAL}

La prescripción nutricional habitual de los pacientes estudiados, consideraba una dieta hiperproteica, debido al estado hipercatabólico derivado del tipo de patología presente. No obstante, por la influencia de la ingesta proteica en el resultado del examen de medición del nitrógeno ureico en orina de 24 horas, se solicitó al nutricionista a cargo de los pacientes, la planificación de una dieta con bajo aporte proteico el día previo al examen, lo cual fue ratificado mediante la aplicación y evaluación de una encuesta recordatoria de 24 horas.

\section{DETERMINACIÓN DEL CATABOLISMO PROTEICO}

La excreción urinaria de nitrógeno ureico, el cual es considerado un indicador fiable de la vertiente catabólica del metabolismo celular y tisular, en particular del recambio proteico (16), fue empleada en la investigación para estimar la tasa de catabolismo y el requerimiento proteico.

Luego de descartar en los pacientes la presencia de enfermedad renal, y con indicación de dieta hipoproteica el día anterior al examen (< 40 g/día), se evaluó el catabolismo proteico mediante la mediación nitrógeno ureico en orina (NUU) de 24 horas. El requerimiento proteico se determinó mediante la fórmula: (NUU de 24 horas $(\mathrm{g})+4$ otras pérdidas nitrogenadas $(\mathrm{g})) \times 6,25$. El valor $4 \mathrm{~g}$ agregado al NUU, representó una estimación liberal de la pérdida no medida de NUU, correspondiente al sudor, pelo, piel y heces. El factor 6,25 se asoció a que el nitrógeno representa al 16\% (es decir 1/6,25) del peso de la proteína de la dieta (17).

Se clasificó el grado del estado catabólico, según NUU de 24 horas 17:

\begin{tabular}{ll} 
Grado de catabolismo & NUU en $24 \mathrm{~h}$ \\
\hline Normal & $<5 \mathrm{~g}$ \\
Leve & $5-10 \mathrm{~g}$ \\
Moderado & $10-15 \mathrm{~g}$ \\
Grave & $>15 \mathrm{~g}$
\end{tabular}

\section{DETERMINACIÓN DE LA TASA METABÓLICA EN REPOSO POR CALORIMETRÍA INDIRECTA}

En la investigación se utilizó el método de la calorimetría indirecta, el cual ha sido ampliamente considerado como uno de los más precisos en la determinación de la tasa metabólica en reposo, en pacientes hospitalizados (18).

La medición de la tasa metabólica en reposo mediante calorimetría indirecta, (TMR CI) utilizó un equipo Datex Ohweda modelo Deltatrac II, perteneciente a la Unidad de Metabolismo Energético de la Universidad del Bío- Bío. A nivel ambiental se controló que la concentración de $\mathrm{CO}_{2}$ fuera menor a $3 \%$ y existiera termo neutralidad ambiental $\left(20-24^{\circ} \mathrm{C}\right)$. El equipo fue auto calibrado antes de cada medición, utilizando una mezcla de gases de $95 \% \mathrm{O}_{2}$ y $5 \% \mathrm{CO}_{2}$. Previo a la medición de la TMR Cl, se verificó ausencia de antecedentes de enfermedad tiroidea, ayuno previo (10-12 h), anemia y dolor; también se controlaron los siguientes signos vitales: temperatura corporal a nivel axilar menor a $37.0{ }^{\circ} \mathrm{C}$, y una frecuencia respiratoria de 12-18 respiraciones por minuto. Todas las mediciones fueron realizadas a las 8:30 h AM, con el paciente en posición supina, sin dormir y tranquilo. Las mediciones se efectuaron una vez que el sujeto alcanzó el estado estable con un coeficiente de variación intrasujeto de $\leq 10 \%$ en la medición del consumo de oxígeno $\left(\mathrm{VO}_{2}\right)$ y dióxido de carbono $\left(\mathrm{CO}_{2}\right)$. Toda indicación de analgésicos en los pacientes, fue administrado después de la medición del examen de calorimetría indirecta. La validez del examen se ratificó mediante el valor del cociente respiratorio (Qr), obtenido de la relación entre el $\mathrm{CO}_{2}$ eliminado y el $\mathrm{O}_{2}$ consumido por el paciente, el que debió mostrar un rango filológico normal de 0,7-1,0 (19); como también mediante la verificación de la fluctuación en el intercambio de volumen de $\mathrm{CO}_{2}(\mathrm{ml} / \mathrm{min}$.) y de $\mathrm{O}_{2},(\mathrm{ml} / \mathrm{min})(20)$.

Posteriormente los resultados de TMR Cl y NUU de 24 
de los pacientes, fueron comparados entre las patologías estudiadas.

\section{GASTO ENERGÉTICO TOTAL}

El gasto energético total (GET) se obtuvo multiplicando la TMR CI por el tipo de reposo físico indicado; en el reposo relativo se utilizó el factor $1,3 \mathrm{y}$, en el reposo absoluto, el factor 1,2 (20).

\section{CÁLCULO DEL FACTOR TRAUMA}

El factor trauma que determina el porcentaje de aumento de la TMR respecto a la condición de normalidad, se calculó a través de la TMR Cl dividido por la tasa metabólica en reposo, calculada a través de la fórmula Harris Benedict (TMB HB) $(21,2)$.

\section{ANÁLISIS ESTADÍSTICO}

El tamaño mínimo de la muestra ( $n=7$ para cada patología), se determinó utilizando las diferencias estimadas en el porcentaje de aumento de TMR, correspondiente a 1,13 $\pm 0,12$ $D^{2}, 1,64 \pm 0,09 D^{2}$ en pancreatitis aguda y quemadura, respectivamente, con una potencia de 0,8 y una significación del $5 \%$.

Se determinó el ajuste de la distribución normal de las variables continuas a través del test de Shapiro- Wilk.

La comparación de las variables en estudio según patología para ambos géneros, se realizó mediante la prueba de la mediana dado el rango de los datos donde el promedio no fue el mejor representante para la distribución.Todos los test realizados fueron del tipo no paramétrico, debido a los tamaños de muestra de los distintos grupos comparativos. El nivel de significación fue 5\% y todos los análisis se realizaron utilizando el software Stata versión 10.0.

\section{RESULTADOS}

La muestra estuvo representada en un $27,8 \%$ por mujeres y en un $72,2 \%$ por hombres.
En los pacientes con pancreatitis aguda, los varones presentaron un IMC promedio dentro del rango de normalidad de $24,74 \pm 1,15$ DS, a diferencia de las mujeres donde el promedio fue clasificado de obesidad 32,04 + 5,03 DS; concordante con el valor promedio de grasa corporal, el cual fue superior al $30 \%$, porcentaje determinante de obesidad en la mujer (22). La distribución porcentual respecto a la clasificación del estado nutricional según IMC fue: normal en un $25 \%$, sobrepeso en un $50 \%$ y de obesidad en un $25 \%$.

Respecto al IMC, los hombres y las mujeres con quemaduras menores mostraron un valor promedio en la clasificación del estado nutricional de sobrepeso. En cuanto a la composición corporal, los hombres en promedio presentaron un porcentaje de grasa corporal normal, a diferencia de las mujeres donde fue superior a lo aceptable (24). La distribución porcentual con respecto a la clasificación del estado nutricional según IMC fue: normal en un $50 \%$, sobrepeso en un $40 \%$ y de obesidad en un $10 \%$ (tabla 1).

El estudio de la TMR Cl y GET según patología, no mostró diferencias significativas entre las patologías y según género ( $p>0,05)$ (tabla 2).

El factor trauma (TMR CI/TMB HB), no resultó ser estadísticamente diferente por patologías $(P=0,506)$, cuyo valor promedio en las patologías estudiadas fue 0,91 \pm 0,14; menor en aproximadamente $9 \%$ a lo estimando mediante la fórmula Harris Benedict.

El valor promedio del QR en los pacientes con pancreatitis aguda fue $0,75 \pm 0,0$ y en los pacientes quemados $0,87 \pm 0,1$, presentando diferencias estadísticamente significativas según el test de Kruskal Wallis $(p=0,029)$.

El estudio del catabolismo proteico mostró en ambas patologías un catabolismo leve, sin diferencia estadísticamente significativa $(p>0,05)$ (tabla 3$)$. La mediana del requerimiento proteico fue de 1,19 g/Kg/día, también sin diferencia estadísticamente significativa entre las patologías estudiadas $(p>0,05)$ (tabla 4).

\section{TABLA 1}

Parámetros antropométricos

$\begin{array}{ll}\text { Patología } & \text { Variable } \\ & \mathrm{N} \\ \text { Pancreatitis } & \text { Edad (años) } \\ \text { aguda } & \text { Talla (mt) } \\ & \text { Peso (kg) } \\ & \text { Índice de Masa Corporal }\left(\mathrm{Kg} / \mathrm{m}^{2}\right) \\ & \text { Masa grasa (\%) } \\ & \text { Masa libre de grasa (\%) } \\ & \mathrm{N} \\ & \text { Superfície corporal quemada (\%) } \\ & \text { Edad (años) } \\ & \text { Talla (mts) } \\ & \text { Peso (kg) } \\ \left.\text { Quemadice de masa corporal (Kg/m }{ }^{2}\right) \\ \\ \text { Masa grasa (\%) } \\ \text { Masa libre de grasa (\%) }\end{array}$

$$
\begin{gathered}
\text { Hombre } \\
\bar{x} \pm s
\end{gathered}
$$

5

$$
\begin{gathered}
45,20 \pm 13,17 \\
1,72 \pm 0,07 \\
73,48 \pm 5,54 \\
24,74 \pm 1,15 \\
20,18 \pm 2,79 \\
79,82 \pm 2,79
\end{gathered}
$$

8

$$
\begin{gathered}
4,37 \pm 3,29 \\
52,12 \pm 13,37 \\
1,66 \pm 0,06 \\
70,26 \pm 14,53 \\
25,35 \pm 5,00 \\
20,28 \pm 6,21 \\
79,72 \pm 6,21
\end{gathered}
$$

Género

$$
\begin{gathered}
\text { Mujer } \\
\bar{x} \pm s
\end{gathered}
$$

3

$40,00 \pm 12,16$

$1,54 \pm 0,07$

$76,27 \pm 17,90$

$32,04 \pm 5,03$

$33,53 \pm 2,43$

$66,47 \pm 2,43$

2

$4,0 \pm 0,00$

$43,00 \pm 5,65$

$1,51 \pm 0,05$

$62,75 \pm 7,42$

$27,55 \pm 1,34$

$33,85 \pm 4,59$

$66,15 \pm 4,59$ 


\section{DISCUSIÓN}

Estados patológicos tales como, quemadura y pancreatitis aguda, provocan en los pacientes un estado hiper metabólico $(1,2)$ y pérdida de la masa muscular (3).

En la investigación la TMR CI y el GET día, resultó similar $(p>0,05)$ entre las patologías estudiadas. El GET mostró un requerimiento energético promedio en ambas patologías de $24 \mathrm{cal} / \mathrm{Kg}$ peso/día, inferior al rango de 30-40 Kcal/Kg peso/ día, habitualmente recomendado a los pacientes con patologías asociadas a estados híper metabólicos (23). La disparidad numérica según género, imposibilitó la comparación entre grupos y estados patológicos, sin embargo, la mayor representación del género masculino en la muestra estudiada, y su mayor nivel de masa muscular, pudo haber influido en los resultados encontrados.

Entre los factores descritos como determinantes de la variación de la TMR en estados patológico, se encuentran: la fase de la enfermedad (aguda o de recuperación), agresividad de la patología, el tratamiento médico y la presencia de malnutrición previa (4). Para evitar que los resultados encontrados fueran explicados por la fase del curso de la enfermedad, se consideró en la injuria la existencia de dos fases diferentes desde un punto de vista fisiopatológico: primero, la denominada fase de Reflujo, que comprende alrededor de las 24 horas posterior al trauma, donde el paciente cursa con un estado hipo metabólico; seguido de la fase siguiente Ilamada Flujo, caracterizada por un estado híper metabólico (3), que alcanza su máximo nivel al cuarto día de iniciada la injuria, para luego decaer hasta un nivel basal del séptimo a décimo día. En la investigación se aceptó realizar en los pacientes quemados las mediciones de la TMR Cl, también en días posteriores a lo señalado, en consideración a que se ha descrito estados hipermetabólicos persistentes, aún durante la fase de convalecencia (24).

Respecto a la agresividad de la patología y su influencia en la TMR, el bajo porcentaje en promedio de SCQ $(<5 \%)$ de los pacientes quemados, podría explicar la baja TMB Cl encontrada. En relación a lo anterior, se ha definido una correlación positiva entre la TMR y el porcentaje de la superficie corporal quemada, señalándose en quemaduras cercanas al $10 \%$ de SCQ, sólo leves aumentos en la TMR, que oscilan entre 0 y $10 \%$, aproximadamente (25); rango muy inferior a lo considerado rutinariamente en la nutrición clínica.

En los pacientes con pancreatitis aguda se ha descrito aumentos en la TMR que varían en $13-14 \%(2,26)$, lo cual en el estudio no fue observado, posiblemente por el bajo índice de severidad registrado en la mayoría de los pacientes estudiados.

Debido al conocido efecto de algunos medicamentos tales como: sedantes, analgésicos, beta bloqueador y relajante muscular, en la disminución de la TMR (27), todos los medicamentos consumidos por los pacientes estudiados fueron administrados en un horario posterior a la realización del examen de calorimetría indirecta. Sin embargo, no es posible descartar totalmente el efecto en la TMR de aquellos administrados el día previo.

El análisis del estado nutricional de la muestra arrojó ausencia de pacientes enflaquecidos, permitiendo también descartar la influencia de un estado hipo metabólico asociado a desnutrición calórica-proteica (28).

Otro factor que contribuye a disminuir la TMR específicamente en los pacientes quemados, es la termo neutralidad ambiental, condición que estuvo presente en las salas clínicas donde ellos permanecían (29).

TABLA 2

Tasa metabólica en reposo medido por calorimetría indirecta y gasto energético total, según patología y género.

\begin{tabular}{|c|c|c|c|c|}
\hline Patología & \multicolumn{2}{|c|}{ Tasa metabólica en reposo (Kcal/día) } & \multicolumn{2}{|c|}{$\begin{array}{l}\text { Gasto energético total (Kcal/Kg/día) } \\
\text { (TMR CI x Factor actividad física) }\end{array}$} \\
\hline \multirow[t]{2}{*}{ Género } & $\begin{array}{c}\text { Hombres }(H) \\
(n=13)\end{array}$ & $\begin{array}{l}\text { Mujeres }(M) \\
\quad(n=5)\end{array}$ & $\begin{array}{l}\text { Hombres }(H) \\
(n=13)\end{array}$ & $\begin{array}{c}\text { Mujeres }(M) \\
\quad(n=5)\end{array}$ \\
\hline & $M e R_{e}=x_{\max }-x_{\min }$ & $M e R_{e}=x_{\max }-x_{\min }$ & $M e R_{e}=x_{\max }-x_{\min }$ & $M e R_{e}=x_{\max }-x_{\min }$ \\
\hline Pancreatitis aguda & $1.680(1175-1825)$ & $1.125(1070-1345)$ & $27.4(21.4-32.1)$ & $21.4(18.0-21.9)$ \\
\hline Quemadura & 1.425 (1150-1510) & 1.115 (1110-1120) & $24.9(19.2-29.8)$ & $21.5 \quad(19.6-23.4)$ \\
\hline
\end{tabular}

Prueba mediana hombres $p=0,284$

Prueba mediana mujeres $p=0,660$

Prueba de la mediana hombres $p=0,592$

Prueba mediana mujeres $p=0,925$

TABLA 3

Grado de catabolismo proteico, según patología

Patología

Quemadura

Pancreatitis aguda
$\operatorname{NUU}(\mathrm{g} / 24 \mathrm{~h})$

Mediana

8,91

9,11
Clasificación de grado catabólico

Leve

Leve

Test Wilcoxon. Mann.Whitney $p>0,05$ 
El factor trauma promedio en las patologías estudiadas fue de 0,91 ( $p>0,05)$, siendo la TMR Cl de los pacientes estudiados, menor en un $9 \%$ a lo calculado a través de la fórmula Harris Benedict. Esta fórmula tiende a sobrestimar la tasa metabólica en reposo en personas sanas como enfermas, lo que puede explicar el hipometabolismo observado (30). Respecto a la pancreatitis, cabe mencionar que también se ha descrito una alta dispersión en el factor trauma (0.67-1.7) en los pacientes con pancreatitis aguda severas y leves (31).

La oxidación preferente de los sustratos energéticos evaluada mediante el cociente respiratorio (32), resultó ser diferente estadísticamente $(p=0,029)$. Sin embargo, debido a la baja sensibilidad y especificidad descrita (19), no fue utilizado como indicador de sobrealimentación o subalimentación en los paciente analizados.

La determinación de los requerimientos proteicos de los pacientes mediante NUU, mostró en ambas patologías un estado de hipercatabolismo leve sin diferencias estadísticamente significativas $(p>0,05)$, resultando una mediana en el requerimiento proteico de $1,19 \mathrm{~g} / \mathrm{Kg} /$ día, levemente superior al rango considerado normal en clínica $(0,8-1,0 \mathrm{~g} /$ $\mathrm{kg} /$ día) $(33,34)$. El requerimiento proteico en los pacientes quemados fue inferior a lo recomendado de 1,5-2.0 g/ $\mathrm{kg} / \mathrm{día}$ $(35,36)$, y para los pacientes con pancreatitis aguda, estuvo dentro de lo sugerido de 1,0-1,5 g/Kg/día (37). Los pacientes con quemaduras leves presentaron una mediana en el NUU de $8,91 \mathrm{~g} / 24 \mathrm{~h}$, inferior a lo encontrado en otro estudio $(11,1 \pm 0,7$ g), el cual además mostró una relación directa entre el tamaño de la quemadura y la ureagénesis (38).

En pancreatitis aguda no hay investigaciones que relacionen la severidad con la pérdida de NUU. El 62,5\% de los pacientes con pancreatitis aguda clasificó con un índice de severidad bajo, según el criterio de Baltazar, presentando la muestra de manera general, una mediana de NUU de 9,11g/ día, lo cual fue esperablemente menor a lo reportado en pancreatitis agudas severas (20-40 g/día.) $(39,40)$.

En conclusión, los requerimientos energéticos y proteicos de los pacientes estudiados con quemaduras menores y pancreatitis agudas, fueron similares, y menor a lo esperado, lo cual puede ser explicado principalmente por la menor severidad de los estados patológicos observados, rechazándose por lo tanto la hipótesis del estudio, la cual señalaba que el requerimiento energético y proteico, es mayor en el paciente quemado.

\section{RESUMEN}

Los estados patológicos de quemadura y pancreatitis aguda, se asocian a estados híper metabólicos e híper catabólicos. El objetivo de este estudio fue determinar los requerimientos energéticos y proteicos mediante un estudio analítico transversal en 18 pacientes: 10 con quemadura menores al 5\% de la superficie corporal quemada y 8 con pancreatitis aguda. Se determinó el peso, la talla, el IMC, los pliegues cutáneos, la tasa metabólica en reposo por calorimetría indirecta (TMR $\mathrm{Cl}$ ), el catabolismo proteico mediante nitrógeno ureico (NUU) orina de 24 horas, factor trauma (TMR CI/TMB fórmula Harris Benedict). La muestra estuvo representada en $72,2 \%$ por hombres y $27,8 \%$ por mujeres. El estudio en pacientes quemados o con pancreatitis aguda se realizó en promedio a los $16,1 \pm 13,04$ DS y 8,8 $\pm 2,0$ DS días posteriores al inicio de la enfermedad, respectivamente. No mostró diferencias estadísticamente significativas en $T M R C I$, resultando un requerimiento energético promedio de $24,4 \mathrm{kcal} / \mathrm{Kg} /$ día y $23,2 \mathrm{kcal} / \mathrm{Kg} /$ día, en pacientes con quemadura y pancreatitis aguda, respectivamente, en ambos géneros ( $p>0,05)$; tampoco en el gasto energético total (GET) ( $p>0,05)$. Los pacientes con pancreatitis aguda o quemadura presentaron un catabolismo leve y su requerimiento proteico fue $1,19 \mathrm{~g} / \mathrm{Kg} /$ día $(p>0,05)$. En los pacientes con pancreatitis aguda el factor trauma fue 0,91 ( $p>0,05$ ). En conclusión, en ambas patologías estudiadas el requerimiento energético de los pacientes fue inferior a lo reportado, respecto al requerimiento proteico, siendo menor en el paciente quemado.

Palabras clave: Requerimiento proteico, nitrógeno ureico urinario, pancreatitis aguda, quemadura, requerimiento energético.

Agradecimientos: La autora agradece al Director Médico del Hospital Clínico Herminda Martín de Chillán, Dr. Rodrigo Avendaño Brandeis, por haber facilitado la realización de la investigación, y al doctor Erik Díaz Bustos por su asesoría técnica.

\section{BIBLIOGRAFÍA}

1. Long Cl, Shaffel N, Geiger JW, et al. Metabolic response to injury and illness: estimation of energy and protein needs from indirect calorimetry and nitrogen balance. JPEN. 1979; 3: 452-6.

2. Barak N, Wall-Alonso E, Sitrin MD. Evaluation of stress factors and body weight adjustments currently used to

TABLA 4

Requerimientos de proteínas (g/Kg/día), según patología

\begin{tabular}{lccc}
\hline \multirow{2}{*}{ Patología } & Hombre & Género & Mujer \\
& Mediana & Mediana & Total \\
& $n=5$ & $\mathrm{n}=3$ & 1,24 \\
Pancreatitis & 1,32 & 0,55 & $\mathrm{n}=10$ \\
& $\mathrm{n}=8$ & 1,18 & 1,19 \\
Quemadura & 1,19 & $\mathrm{n}=5$ & $\mathrm{n}=18$ \\
& $\mathrm{n}=13$ & 0,77 & 1,19 \\
\hline
\end{tabular}


estimate energy expenditure in hospitalized patients. JPEN 2002; 26:231-8.

3. M Shils. Nutrition en Salud y Enfermedad En: El estado hipercatabólico. $9^{\circ}$ Edición, México, Editorial Mc. 2002.p 1805-20.

4. Elia M. Insight into energy requeriments in disease. Public Health Nutr. 2005; 8: 1037-52.

5. Wang Z, Heshka S, Heymsfield SB, Shen W, Gallagher D. A cellullar -level approach to predicting restinga energy expenditure across the adult years. Am J Clin Nutr. 2005; 81: 799- 806.

6. Ortiz C, Gómez-Tello V, Arbeloa S. Requerimientos de macronutrientes y micronutrientes. Nutr Hosp. 2005; 2:13-7.

7. Balthazar E, Robinson D, Megibow A. Acute pancreatitis: value of CT in establishing prognosis. Radiology. 1990; 174: 331- 6.

8. Schwaner J, Rivas F, Cancino A, Torres O, Briceño $C$, Riquelme P. Pancreatitis aguda: Indice de severidad en tc. Evaluación de complicaciones y hospitalización. Rev Chil Radiol. 2003; 9: 187- 93.

9. Schwartz R J, Chirino CN, Saenz SV y Rodriguez TV. Algunos aspectos del manejo del pacienter quemado en un servicio de cirugía infantil. A propósito de 47 pacientes pediátricos. Ila parte. Rev Argent Dermatol. 2008; 89: 165-73.

10. Gordon $C$, Cameron $W$, Rochet $A$. Stature, recumbent lenght, and weight. In: Lohman T, Roche A, Martorell R. Anthropometric standardization reference manual. Abidged Edition. 1988:p 3-8.

11. Callaway W, Cameron, Bouchard C, Himes J, Lohman T, Martín $A$, et al. Circumferences. In: Lohman $T$, Roche $A$, Martorell R. Anthropometric standardization reference manual. Abidged Edition. 1988: p 39-54.

12. Harrison G, Buskirk E, Carter J, Johnston F, Lohman $T$, Pollock $M$, et al. Skinfold thicknesses and measurement technique. In: Lohman T, Roche A, Martorell R. Anthropometric standardization reference manual. Abidged Edition. 1988: $p$ 55-70.

13. Durnin, JV, Womersley, J. Body fat assessed from total body density and its estimation from skinfold thickness: measurements on 481 men and women aged from 16 to 72 years. Br J Nutr. 1974; 32: p 77-97.

14. Adaptado de The ASPEN Nutrition Support Practice Manual. American Society of Enteral and Parenteral Nutrition. Silver Sring, 1998.

15. Burge JC. Obesidad. En: Matarese L, Gottschlich M. Nutrición Clínica Práctica. Editorial Elsevier. 606-11. España, Madrid. 2004.

16. Alcock NW. Laboratory tests for assessing nutritional status. En: Modern Nutrition in Health and Disease(Editores: Shils $M E$, Olson JA, Shike M, Ross AC). Novena Edición. Williams \&Wilkins. Baltimore, MD: 1999: p 923-35.

17. Morgan S, Weinsier R. Valoración Nutricional. En: Nutrición Clínica. 2a ed.España, Ediciones Harcourt, 2000: p 182-94.

18. Cooney RN, Frankenfield DC. Determining energy needs in critically ill patients: equations or indirect calorimeters. Curr Opin Crit Care. 2012;18(2):174-7.

19. McClave SA, Lowen CC, Kleber MJ, McConnell JW, Jung $L Y$, Goldsmith LJ. Clinical use of the respiratory quotient obtained from indirect calorimetry. JPEN 2003; 27: 21-6.

20. Boullata J,Williamns J, Cottrell F at el. Accurate Determination of energy need in hospitalizad patient. J Am Diet Assoc. 2007; 107:393-401.

21. Harris JA, Benedict FG. Biometric Study of Basal Metabo- lism in Man. Washington, DC: Carnegie Institute, 1919: (Publication 279), p 40-4.

22. Definitions and proponed current classifications of obesity. Bray G, Bouchard C, James WPT. Handbook of obesity. New York Marcel Dekker. 1998, p 31-40.

23. Olveira G, Gonzalo M. Requerimientos nutricionales. En: Manual de nutrición y metabolismo. España. Ediciones Díaz de Santos. 2006.p 25-47.

24. Elia M. Hunger disease: Metabolic response to starvation, injury and sepsis. Clin Nutr. 2000; 19:379-86.

25. Elia M. Artificial feeding: requeriments and complications. Med Internac. 1994; 22: 411-5.

26. Morgan S, Weinsier R. Nutrición y páncreas. En: Nutrición Clínica. $2^{a}$ ed.España, Ediciones Harcourt, 2000: p 226-7.

27. Roland N Dickerson, Pharm D, Lori Roth-Yousey. Medication effects on metabolic rate: A systematic Review (part 1) J Am Diet Assoc. 2005; 105:835-43.

28. Torum B, Chew F. Desnutrición calórica proteíca. Shils E, Maurece. Nutrición en la Salud y la Enfermedad. 9 na Edición Edit. Mc Graw - Hill Interamericana. España. 2002.

29. Prelack K, Dylewski M, Sheridan R. Practical guidelines for nutritional management of burn injury and recovery. Burns. 2007; 33:14-24.

30. Frankenfield D, Roth-Yousey-L, Compher C. Comparison of Predictive Equations for Resting Metabolic Rate in Healthy Nonoobese and Obese Adults: A Systematic Review. J Am Diet Assoc. 2005;105:775-89.

31. Velasco N, Papapietro K, Rapaport J, Guzmán S, Maíz A et al. Variability of measured energy expenditure in patients with acute panbcreatitis: is it posible to obtain a reliable pathology factor for these cases?. Rev Med Chil. 1994; 122:48-52.

32. Frankefield D. Dinámica Energética EN: Nutrición Clínica Práctica Por Matarese L., Gottschlich M., 2a ed. España, Editorial Elsevier Sanders. 2004, p. 85-6.

33. Energy and protein requirements. Report of a Joint FAO/ WHO/UNU ExpertConsultation. Geneva, World Health Organization, (WHO Technical Report Series, No. 724). 1985.

34. Morgan S, Weinsier R. Valoración Nutricional. En: Nutrición Clínica. $2^{a}$ ed.España, Ediciones Harcourt, 2000: 195-213.

35. Prelack K, Dylewski M, Sheridan RL. Practical guidelines for nutritional management of burn injury and recovery. Burns. 2007; 33(1):14-24.

36. Williams FN, Branski LK, Jeschke MG, Herndon DN. What, how, and how much should patients with burns be fed? Surg Clin North Am. 2011; 91(3):609-29.

37. García Almansa A, García Peris P. Tratamiento nutricional de los enfermos con pancreatitis aguda: cuando el pasado es presente. Nutr Hosp. 2008; 23(2):52-8.

38. Kagan $R$, Matsuda T, Hanumadass M, Castillo B, Jonasson $O$. The effect of burn wound size on ureagenesis and nitrogen balance. Ann Surg. 1982; 195:70-4.

39. Gupta R, K Patel, Calder PC, Yaqoob P, JN Primrose, Johnson $C D$. A randomized clinical trial to assess the effect of total enteral and total parenteral nutriotional support on metabolic, inflammatory and oxidative markers in patients with predicted severe acute pancreatitis (APACHE // > or = 6). Pancreatology. 2003; 3: 406-13.

40. Shaw JH, Wolfe RR. Glucose, fatty acid, and urea kinetics in patients with severe pancreatitis. The response to substrate infusion and total parenteral nutrition. Ann Surg. 1986; $204: 665-72$. 